\title{
Effect of Psychological Nursing on the Mental State and Quality of Life of Patients After Heart Valve Replacement for Rheumatic Heart Disease
}

\author{
Lijuan Wu*, Liping Feng, Hui Chen \\ Department of Cardiothoracic Surgery, Zhenjiang First People's Hospital, Zhenjiang 212000, Jiangsu Province, China \\ *Corresponding author: Lijuan Wu, 498680525@qq.com
}

\begin{abstract}
Objective: To explore the effect of psychological nursing on the mental state and quality of life of patients after heart valve replacement for rheumatic heart disease. Methods: Seventy-four patients with rheumatic heart disease that underwent heart valve replacement in Zhenjiang First People's Hospital from January 2017 to May 2020 were selected as the research subjects. Thirty-seven patients that were treated from January 2017 to September 2018 were included in the control group. Routine care was provided for the patients in the control group. From October 2018 to May 2020, the Department of Cardiothoracic Surgery improved their preoperative and postoperative psychological care. Thirty-seven patients that were treated during this period were included in the research group. The research group received psychological nursing care in addition to routine care. The effects of different nursing modes on the quality of life and postoperative mental state of the patients were determined. Results: The scores for depression, anxiety, somatization, and compulsion in the research group were significantly lower than those in the control group $(p<0.05)$; the scores for the quality of life of patients in the research group were significantly higher than those in the control group $(p<0.05)$. Conclusions: Strengthening psychological nursing for patients with rheumatic heart disease is helpful to improve their quality of life and mental state after heart valve replacement.
\end{abstract}

Keywords: Psychological nursing; Rheumatic heart disease; Heart valve replacement; Quality of life

Publication date: September 2021; Online publication: September 30, 2021

\section{Introduction}

Surgical treatment is the most important treatment for rheumatic heart disease, among which heart valve replacement is the most common type of heart surgery ${ }^{[1]}$. In the clinical practice of nursing patients after heart valve replacement for rheumatic heart disease, it has been found that the curative effect of heart valve surgery is acceptable and excellent results have been achieved in improving patients' cardiopulmonary function and exercise capacity. However, the early postoperative mental state and quality of life of patients, which are important factors that affect the prognosis, have always been an important topic in clinical nursing. In this study, psychological nursing was applied during perioperative nursing for patients after heart valve replacement for rheumatic heart disease in order to improve the mental state and overall quality of life of patients.

\section{Data and methods}

\subsection{General information}

Seventy-four patients with rheumatic heart disease that underwent heart valve replacement in Zhenjiang First People's Hospital from January 2017 to May 2020 were selected as the research subjects. Thirty-seven 
patients that were treated from January 2017 to September 2018 were included in the control group. From October 2018 to May 2020, the Department of Cardiothoracic Surgery improved their preoperative and postoperative psychological care. Thirty-seven patients that were treated during that period were included in the research group. The research group comprised of 15 male patients and 22 female patients; age ranging from 41 years old to 67 years old, with a mean age of $52.86 \pm 2.37 ; 26$ cases with grade II cardiac function and 11 cases with grade III cardiac function; 21 cases of single valve replacement and 16 cases of double valve replacement. The control group comprised of 17 male patients and 20 female patients; age ranging from 39 years old to 68 years old, with an average age of $52.17 \pm 2.49 ; 28$ cases with grade II cardiac function and 9 cases with grade III cardiac function; 23 cases of single valve replacement and 14 cases of double valve replacement.

The inclusion criteria were patients that met the surgical indications without any contraindications related to heart valve replacement. The exclusion criteria were patients with cognitive impairment ${ }^{[2]}$, patients that were unable to communicate normally due to language, mental, or hearing impairment, and patients with malignant tumors or with liver, kidney, or respiratory dysfunction. There was no significant difference between the two groups $(p>0.05)$.

\subsection{Nursing methods}

The control group received routine nursing care, which included admission education, nursing according to doctor's advice, discharge guidance, etc. However, psychological nursing was added for the patients in the research group. First, psychological nursing was provided preoperatively. Preoperative patients often have worries and fears. Nurses should observe and evaluate the psychological state of patients as well as provide appropriate psychological nursing interventions to reduce the psychological pressure faced by the patients so as to not affect the curative effect of the surgery. The measures that can be taken include communicating with patients, understanding the causes of the worries and fears, as well as conducting targeted psychological counseling. If patients do not understand the worries caused by the surgery, nurses should explain the process of the surgery, intraoperative cooperation, and postoperative precautions; guide the patients to view the surgery with a correct attitude and modify their preoperative psychological state. Nurses can also communicate with patients' family members, encourage them to give more support through actions and words, as well as strengthen the feelings of patients in regard to family support, thus reducing preoperative anxiety and panic among patients ${ }^{[3]}$. Second, postoperative psychological nursing was also provided. Postoperative patients often have limited activity and experience chest pain. In this state, patients would tend to develop negative emotions. Due to the pain at the surgical site, postoperative training compliance would reduce significantly, and some patients do not even dare to cough. This is not conducive to the rehabilitation of patients. Therefore, nurses should reinforce nursing guidance and provide psychological comfort to patients. Nursing guidance includes teaching patients abdominal breathing and effective coughing methods so as to avoid sputum accumulation, which increases the risk of lung infection, informing patients about the importance of rehabilitation training for the recovery of heart and lung function, as well as encouraging them to actively cooperate with the training. At the same time, music relaxation training, music therapy, and mindfulness decompression intervention can also be used to reduce the feeling of pain. If necessary, sedative and analgesic drugs can be used according to the doctor's prescription.

\subsection{Observation indicators}

(1) Postoperative mental state: Symptom Checklist-90 (SCL-90) was used. This checklist included 9 evaluation factors: depression, anxiety, somatization, compulsion, paranoia, hostility, terror, interpersonal sensitivity, and psychoticism. Finally, the scores for the positive items and the total 
score were calculated. The higher the score, the better the mental state of the patient.

(2) Quality of life: 36-Item Short Form Survey (SF-36) was used to evaluate the quality of life of the patients from the two groups at discharge. The evaluation dimensions include life vitality, mental health, physiological function, social function, physical pain, overall health, emotional function, physiological function, and total score. The higher the score, the better the patient's quality of life.

\subsection{Statistical analysis}

Statistical Package for the Social Sciences (SPSS) version 24.0 statistical software was used to process the data. The counting data were expressed in $(\mathrm{n} / \%), \chi^{2}$ test, and the measurement data were expressed in $(\bar{x} \pm$ $\mathrm{s}), \mathrm{t}$ test. The difference is statistically significant if $p<0.05$.

\section{Results}

\subsection{Multidimensional comparison of the postoperative mental state between the two groups}

The mental state scores, average scores, and total scores of the patients in the research group were significantly lower than those in the control group $(p<0.05)$ (Table 1).

Table 1. Comparison of the multiple dimensions of postoperative mental state between the two groups $(\bar{x} \pm$ $\mathrm{s} / \mathrm{min})$

\begin{tabular}{lcccc}
\hline \multicolumn{1}{c}{ Group } & $\begin{array}{c}\text { Research group } \\
(\mathrm{n}=37)\end{array}$ & $\begin{array}{c}\text { Control group } \\
(\mathrm{n}=37)\end{array}$ & T value & $p$ value \\
\hline Depression & $1.94 \pm 0.26$ & $2.37 \pm 0.32$ & 6.344 & $<0.001$ \\
Anxiety & $1.71 \pm 0.19$ & $2.15 \pm 0.25$ & 8.523 & $<0.001$ \\
Somatization & $1.51 \pm 0.37$ & $1.84 \pm 0.52$ & 3.145 & $<0.001$ \\
Compulsion & $2.06 \pm 0.42$ & $2.47 \pm 0.53$ & 3.688 & $<0.001$ \\
Paranoia & $1.63 \pm 0.32$ & $2.07 \pm 0.45$ & 4.847 & $<0.001$ \\
Hostility & $2.04 \pm 0.27$ & $2.55 \pm 0.34$ & 7.145 & $<0.001$ \\
Terror & $1.42 \pm 0.18$ & $1.78 \pm 0.26$ & 6.925 & $<0.001$ \\
Interpersonal sensitivity & $1.98 \pm 0.25$ & $2.43 \pm 0.41$ & 5.700 & $<0.001$ \\
Psychoticism & $1.52 \pm 0.27$ & $1.94 \pm 0.36$ & 5.677 & $<0.001$ \\
Equal share & $1.87 \pm 0.31$ & $2.17 \pm 0.43$ & 3.442 & 0.001 \\
Total score & $154.46 \pm 12.88$ & $193.28 \pm 16.49$ & 11.285 & $<0.001$ \\
\hline
\end{tabular}

\subsection{Multidimensional comparison of the quality of life between the two groups}

The scores for the quality of life of the patients in the research group were significantly higher than those in the control group $(p<0.05)$ (Table 2). 
Table 2. Multidimensional comparison of the quality of life between two groups $(\bar{x} \pm \mathrm{s} / \mathrm{min})$

\begin{tabular}{lcccc}
\hline \multicolumn{1}{c}{ Group } & $\begin{array}{c}\text { Research group } \\
(\mathrm{n}=37)\end{array}$ & $\begin{array}{c}\text { Control group } \\
(\mathrm{n}=37)\end{array}$ & T value & $p$ value \\
\hline Vitality & $80.26 \pm 4.97$ & $71.53 \pm 4.18$ & 8.177 & $<0.001$ \\
Mental health & $69.42 \pm 3.72$ & $62.18 \pm 3.15$ & 9.035 & $<0.001$ \\
Physiological function & $89.15 \pm 4.13$ & $80.26 \pm 3.22$ & 10.326 & $<0.001$ \\
Social function & $79.48 \pm 2.18$ & $72.13 \pm 3.47$ & 10.910 & $<0.001$ \\
Physical pain & $73.53 \pm 2.69$ & $63.54 \pm 5.29$ & 10.239 & $<0.001$ \\
Overall health & $78.64 \pm 3.98$ & $70.82 \pm 3.27$ & 9.234 & $<0.001$ \\
Emotional function & $79.43 \pm 4.11$ & $72.65 \pm 3.22$ & 7.899 & $<0.001$ \\
Physiological function & $75.52 \pm 5.15$ & $64.19 \pm 4.28$ & 10.292 & $<0.001$ \\
Total score & $614.72 \pm 17.43$ & $553.84 \pm 12.79$ & 17.129 & $<0.01$ \\
\hline
\end{tabular}

\section{Discussion}

After surgery for rheumatic heart disease, patients would experience significant trauma, and the rehabilitation cycle often takes a considerable amount of time. Therefore, patients are tend to develop negative emotions, such as sadness, anxiety, and pain. A poor mental state for long term increases the risk of depression and other diseases. It is also a risk factor for other complications, such as myocardial ischemia and arrhythmia, which would seriously affect the rehabilitation of patients' cardiac function. Timely and effective psychological nursing is of great significance to reduce patients' negative emotions and improve their rehabilitation enthusiasm ${ }^{[4,5]}$. Therefore, in this study, psychological nursing was applied in the nursing of patients after their valve replacement for rheumatic heart disease. The results showed that the mental state scores, average scores, and total scores of patients in the research group within the dimensions of depression, anxiety, somatization, and compulsion were significantly lower than those in the control group $(p<0.05)$. The results also showed that the mental state of the patients significantly improved in all dimensions. The is because psychological nursing has improved the compliance of patients and established their confidence to overcome diseases, thus resulting in the stabilization of their blood pressure and heart rate as well as improving the curative effect of the surgery. Careful postoperative nursing guidance enables patients to gain emotional support and introduces various ways for patients to relieve their emotions. This in turn helps to reduce the stress response, psychological burden, and relevant postoperative complications caused by surgery. It also helps to reduce the excitability of the sympathetic nervous system as well as the release of catecholamine in children, thus slowing down the heart rate and reducing the risk of arrhythmia. In addition, the quality of life scores of patients in the research group in regard to various dimensions, such as life vitality, mental health, physiological function, social function, physical pain, overall health, emotional function, physiological function, and total score, were significantly higher than those in the control group $(p<0.05)$, suggesting that the implementation of humanistic care and psychological nursing measures can improve the quality of life of patients after surgery. Preoperative health education deepens patients' understanding of their surgery and helps to reduce their psychological burden. After surgery, its emphasis is on psychological intervention measures, such as mindfulness decompression and music therapy, in order to reduce the postoperative pain experienced by patients. At the same time, it encourages patients to carry out rehabilitation training as soon as possible, laying a solid foundation of improving the quality of life of patients postoperatively.

In conclusion, it is beneficial and worthy of promotion to strengthen psychological nursing for patients with rheumatic heart disease as it helps to improve the quality of life and mental state of these patients after heart valve replacement. 


\section{Disclosure statement}

The authors declare that there is no conflict of interest.

\section{References}

[1] Dai Y, 2019, Anticoagulant Therapy and Nursing of Patients After Mechanical Heart Valve Replacement. Thrombosis and Hemostasis, 25(5): 884-885, 887.

[2] Tai J, Han L, Li Y, et al., 2020, Effect of Health Belief Model on Warfarin Anticoagulation Management in Patients After Mechanical Heart Valve Replacement. Qilu Journal of Nursing, 26(8): 1-5.

[3] Shao Y, Huang F, Zou M, 2019, Effect of Early Rehabilitation Nursing on Complications and Rehabilitation Effect After Heart Valve Replacement. Nursing Practice and Research, 16(22): 104106.

[4] He W, Liao Y, Yin X, et al., 2019, Summary of the Best Evidence of Stage I Cardiac Rehabilitation After Cardiac Valve Replacement. Journal of Nursing, 26(18): 32-36.

[5] Song J, Wang Z, Hu Y, et al., 2019, Effect of Early Exercise Training on Patients After Cardiopulmonary Bypass Heart Valve Replacement. Nursing and Rehabilitation, 18(7): 1-5. 